\title{
Tryptophan Depletion During Continuous CSF Sampling in Healthy Human Subjects
}

\author{
Linda L. Carpenter, M.D., George M. Anderson, Ph.D., Gregory H. Pelton, M.D., \\ Jeffrey A. Gudin, M.D., Paul D. S. Kirwin, M.D., Lawrence H. Price, M.D., \\ George R. Heninger, M.D., and Christopher J. McDougle, M.D.
}

The tryptophan (TRP) depletion paradigm has been employed to investigate mood and behavioral effects of acutely lowering plasma TRP, and presumably brain serotonin (5-hydroxytryptamine [5-HT]) levels through administration of a special diet and/or amino acid drink. Our goal was to test the assumption that a corresponding fall in central levels of TRP and 5-HT (measured by its major metabolite, 5-hydroxyindoleacetic acid [5-HIAA]) occurs during the standard execution of this method in healthy adult subjects. Three males and two females completed the protocol, which included a one-day low-TRP diet and a TRP-free amino acid drink. Lumbar puncture was performed, with placement of an indwelling catheter connected to a peristaltic pump and fraction collector.
Cerebrospinal fluid (CSF) was sampled continuously for a 13.5-hour period (before, during, and after the drink), with fractions removed every 15 minutes. Plasma samples were simultaneously obtained. CSF TRP levels and plasma TRP levels were highly correlated, falling a mean of $92 \%$ and $85 \%$ from baseline, respectively. CSF nadirs were reached several hours after plasma nadirs. CSF 5-HIAA decreased modestly ( $24 \%$ to $40 \%$, mean $31 \%$ change from baseline), with lowest concentrations observed 8-12 hours after the amino acid drink. These data suggest that TRP depletion results in substantial declines in central 5-HT turnover. [Neuropsychopharmacology 19:26-35, 1998] Published by Elsevier Science Inc.
KEY WORDS: Serotonin; Tryptophan; CSF; 5-HIAA; Amino acids

The role of serotonin (5-hydroxytryptamine, 5-HT) in the pathogenesis and treatment of major neuropsychiatric disorders, especially depression, continues to be the subject of intensive research (Maes and Meltzer 1995). One paradigm for studying the role of 5-HT involves acutely manipulating levels of its amino acid precursor, tryp-

From the Butler Hospital, Laboratory for Clinical Neuroscience, Department of Psychiatry and Human Behavior (LLC, LHP), Brown University School of Medicine, Providence, RI; Yale Child Study Center (GMA), Clinical Neuroscience Research Unit, Connecticut Mental Health Center, Department of Psychiatry (GHP, PDSK, GRH), and Department of Anesthesia (JAG), Yale University School of Medicine, New Haven, CT; and Child \& Adolescent Psychiatry (CJM), Indiana University School of Medicine, Indianapolis, IN.

Address correspondence to: Linda L. Carpenter, M.D., Butler Hospital, Laboratory for Clinical Neuroscience, Department of Psychiatry and Human Behavior, Brown University School of Medicine, 345 Blackstone Blvd., Providence, RI, 02906 tophan (TRP), and measuring corresponding behavioral effects. Because the synthesis of 5-HT is dependent on the availability of TRP, rapidly-induced shifts in the central level of TRP should produce transient alterations in the amount of 5-HT available for neurotransmission (Schaechter and Wurtman 1990; Young 1991). Observed or reported changes in behavior or psychiatric symptoms occurring during acute TRP manipulation have provided clues about the mechanism of action of serotonergic drugs, and the biology of several psychiatric disorders.

Beginning in the 1970s, researchers have developed several strategies for altering TRP levels, in efforts to explore the relationship between the precursor amino acid, serotonergic function, and behavior. Preclinical studies demonstrated that dietary restriction of TRP could evoke modest reductions in plasma and brain TRP (Fernstrom 1977), with corresponding behavioral indices reflecting diminished 5-HT function (Lytle et al. 1975; Messing et al. 1976; Gibbons et al. 1979; Moja et al. 
1979; Walters et al. 1979). Dietary TRP restriction in both animals and humans has subsequently been associated with enhanced response to neuroendocrine challenges in a manner consistent with compensatory postsynaptic supersensitivity (Clemens et al. 1980; Delgado et al. 1989), again suggesting that the depletion is diminishing 5-HT function at a central nervous system (CNS) level. More rapid decreases in plasma and brain TRP levels can be achieved by the administration of a TRP-free amino acid mixture (Biggio et al. 1974; Young et al. 1995; Moja et al. 1988). Here the mechanism involves providing substrate for induction of protein synthesis to such a degree that available peripheral TRP stores are largely used up, resulting in a drop in the ratio of TRP to other large neutral amino acids in plasma (Harper et al. 1970). Because TRP competes with the other large neutral amino acids for passage across the blood-brain barrier by an active transport system, a relative TRP depletion is thought to occur in the CNS, essentially paralleling the changes measured in blood (Gessa et al. 1974; PerezCruet et al. 1974). Animal studies of cerebrospinal fluid (CSF) and brain tissue have confirmed that these methods for oral TRP depletion are able to lower CNS levels of TRP, 5-HT, and 5-hydroxyindoleacetic acid (5-HIAA), the major metabolite of 5-HT in the CSF.

While it has not been possible to directly measure central changes in 5-HT in humans (Anderson et al. 1990), decreases of $80-90 \%$ have been observed in peripheral concentrations of TRP following depletion via dietary TRP restriction and/or ingestion of TRP-deficient amino acid mixtures. The decreases have been associated with a number of provocative findings, including rapid and transient worsening of mood, impairments in cognitive function, altered feeding behavior and cocaine craving, changes in pain tolerance, and increases in anxiety or aggressive behavior (Miller et al. 1992). The TRP depletion research paradigm has indeed become a popular tool for investigating the role of serotonergic function in a variety of populations and clinical conditions (e.g., Price et al. $1997 a, b)$. However, the meaningfulness of such findings is predicated on the assumption that central 5-HT functioning is, in fact, substantially reduced by TRP depletion.

This investigation was designed to further test the validity of the TRP depletion paradigm, and to explore the relationship between plasma TRP and central indices of 5-HT by measuring CSF TRP and 5-HIAA, sampled continuously via an indwelling subarachnoid lumbar catheter over a 13.5-hour period before, during, and after, standard TRP depletion in 5 healthy human subjects.

\section{SUBJECTS AND METHODS}

\section{Subjects}

The study was approved by the Human Investigations Committee of the Yale University School of Medicine.
Five adult subjects ( 2 male, 3 female; age $33.6 \pm 4.5$ yrs; range 29-38 yrs) free of psychiatric or medical illness, gave voluntary, written informed consent to participate as paid volunteers in the study. Subjects were accepted if they had no personal or family history of medical, neurologic, or psychiatric illness, and no illicit drug use or regular medication use within the past year. All subjects demonstrated normal values on a battery of screening laboratory tests which included serum chemistries, liver function tests, coagulation indices, complete blood count, thyroid indices, urinalysis and urine toxicology screens, and pregnancy test in females. All subjects demonstrated normal results on physical examination and electrocardiogram before entering the study.

\section{Procedures}

Subjects were admitted to a specialized biological studies suite on the Clinical Neuroscience Research Unit of the Connecticut Mental Health Center, New Haven, CT, for a period of four days. Beginning with breakfast on the first day, subjects were restricted to a $160 \mathrm{mg}$ /day low-TRP diet, which they were instructed to consume in its entirety during day 1 . The diet has been employed extensively in TRP depletion studies at Yale and is described in detail elsewhere (Delgado et al. 1990). Strict bedrest and intravenous fluid therapy (normal saline with $1 \%$ dextrose solution at $250 \mathrm{cc} /$ hour) were initiated at 20:00 $\mathrm{h}$ on the first day and continued through day 3 , to minimize the risk of spinal headache and fasting hypoglycemia during the procedure.

At 08:00 h (day 2), after a night of bedrest and intravenous hydration, subjects underwent lumbar puncture and placement of the subarachnoid catheter, using a modified technique of Bruce and Oldfield (Bruce and Oldfield 1988), as previously described by Geracioti and colleagues $(1992,1993)$. Subjects were placed in the lateral decubitus position in their hospital beds, and after application of intradermal lidocaine anesthesia, an 18-gauge Touhy needle was inserted through the L3-L4 or L4-L5 interspace. After entry into the subarachnoid space a 20-gauge nylon catheter was advanced cephalad 5 to $10 \mathrm{~cm}$, secured externally with Tegaderm tape, and capped. Later, the subarachnoid catheter was extended with sections of sterilized polyethylene and silicon tubing and attached to a peristaltic pump. The total dead space in the system was estimated to be between $0.40-0.45 \mathrm{ml}$. CSF was continuously withdrawn into test tubes by a pump programmed to deliver a flow rate of $0.06 \mathrm{ml} / \mathrm{min}$ (approximately $15-20 \%$ of the normal CSF production rate). The $0.9 \mathrm{ml}$ of CSF collected during each 15 minute time interval was immediately separated into aliquots and frozen on dry ice at the bedside. Approximately two hours were allotted for the subject's adaptation to the indwelling catheter and testing condi- 
tions. Continuous sampling began at 10:30 $\mathrm{h}$ and ended at 24:00 h, with a total volume of $55 \mathrm{cc}$ removed over the 13.5-hour period.

Subjects were permitted to rest in bed, take brief naps, read, converse quietly with visitors or over the phone, or view a television/VCR which was made available in the testing room, during idle time at bedrest. Bedpans and urinals were made available but limited body movement was enforced. Subjects could assume any position lying in bed, provided their heads remained on the pillow. Subjects denied any discomfort from the indwelling catheter once it was in place and taped externally.

At noon on day 2, subjects received the standard TRP-free amino acid mixture (described in detail by Delgado et al., 1990), with proportions of 15 amino acids similar to those found in human milk. The drink was prepared by combining the amino acid powders with enough water to equal a final volume of $350 \mathrm{ml}$, flavored to taste with Hershey's chocolate syrup; more noxious-tasting amino acids were administered as 25 capsules, taken orally prior to the drink. The drink was taken quickly through a straw, and tolerated well by all subjects. To avoid potential effects of fasting, calculation of estimated ingested calories from the amino acid/chocolate mixture, plus energy delivered by the continuous intravenous infusion of dextrose $(0.25 \mathrm{ml} /$ $\mathrm{hr}$ ) were made to ensure stable blood glucose levels throughout the sampling period, without additional meals or oral intake.

Blood samples for plasma TRP and tryosine measurements were drawn before starting the low-TRP diet on day 1 . On day 2, blood samples were collected through a forearm intravenous catheter hourly from 07:00 $\mathrm{h}$ until 11:00 $\mathrm{h}$, and then every 15 minutes during the 13.5-hour period of CSF sampling. A total volume of approximately $310 \mathrm{cc}$ of blood was removed during the study. No blood samples were obtained from one of the five subjects ( 29 year old female), due to limited venous access; the intravenous line established was adequate for administration of fluids but did not permit blood withdrawal.

Behavioral measures included a battery of clinicianand self-ratings of anxiety and mood state, obtained at predetermined time points, every four hours throughout the day of CSF sampling. A trained research nurse clinician, with established reliability on the instruments used, was present throughout the procedure and administered the 25-item Hamilton Depression Rating Scale (HDRS) (Mazure et al. 1986), the Hamilton Anxiety Rating Scale (HARS) (Hamilton 1959), the Beck Depression Inventory (Beck et al. 1961), the 29-item Physical Symptom Checklist (Woods et al. 1986) and visual analog scales (McCormack et al. 1988) describing 16 different mood states.

The subarachnoid catheter was withdrawn at 24:00 $\mathrm{h}$ on day 2 of the study, and subjects remained at bedrest, with continued intravenous hydration, for the subsequent 24 hours. They were allowed to resume eating regular meals ad lib after removal of the subarachnoid catheter. Subjects' heads were gradually elevated while in bed from 12:00 $\mathrm{h}$ to $24: 00 \mathrm{~h}$ on day 3 . Once they were free of side effects and able to ambulate comfortably, they were discharged to home with follow-up phone calls at 24 and 48 hours, and as indicated thereafter.

\section{Hormone and Amino Acid Assays}

Levels of CSF TRP, tyrosine, 5-HIAA, and homovanillic acid (HVA) were determined using a modified version of a previously described high performance liquid chromatographic (HPLC) method (Anderson et al. 1979). These assays were run for all CSF fractions except the first, representing 54 serial samples in each subject. Plasma concentrations of total TRP and tyrosine were determined, after perchloric acid deproteination, using an HPLC-fluorometric method (Anderson et al. 1987). To explore the relationship between TRP and tyrosine, assays were run on all blood samples collected in the first patient. Thereafter, only samples from hourly and halfhourly time points were assayed for measurement of plasma TRP and tyrosine concentrations.

\section{Data Analysis}

Data from the four samples collected within each hour were averaged to identify the baseline and nadir concentrations for CSF TRP and 5-HIAA in each subject. Percent change after amino acid drink was calculated as: 1 -(nadir value divided by baseline value) $\times 100$. Concentrations of CSF TRP, tyrosine, HVA, and 5-HIAA, and plasma TRP levels were analyzed for change over time using a repeated measures analysis of variance (ANOVA). We have previously investigated normal levels of these variables occurring over a 30-hour sampling period with this same collection method, and found no significant underlying diurnal variation or rhythmicity (Kirwin et al. 1997).

The baseline plasma TRP value was determined by the average of two plasma samples drawn before the animo acid drink. Only one pre-diet plasma sample was obtained (drawn at 08:00, day 1). Percent change in plasma TRP after the one-day, low-TRP diet was calculated as 1-(baseline TRP divided by pre-diet TRP) $\times$ 100. Total depletion, attributable to the combination of the diet and the amino acid mixture, was calculated in the same fashion, comparing the nadir value to the prediet value. Plasma levels of TRP were averaged hourly. Pearson's correlation coefficients were generated for comparison of plasma versus CSF TRP values within each of four subjects, and TRP versus tyrosine (both CSF and plasma) in the first subject. 
To test for mood effects from TRP depletion, repeated measures ANOVA was run for HDRS scores over time during the day of sampling. Huyndt-Feld-corrected significance values are reported for all repeated measures ANOVAs where sphericity assumptions were not met.

\section{RESULTS}

\section{Effect of TRP Depletion as Measured by Plasma TRP Levels}

Plasma TRP levels from the single pre-diet time point, baseline, and hourly for the duration of the CSF sampling period, are shown in Figure 1. Ingestion of the low-TRP diet alone produced drops in plasma TRP levels of $16.5 \%$ to $33.6 \%$ (mean, $27.7 \%$ ) from one day to the next. More robust depletion was seen after ingestion of the amino acid mixture; all four subjects for whom plasma data are available reached their TRP nadirs in the sixth hour after the amino acid mixture, with further decreases (baseline to nadir) of $78.4 \%$ to $90.5 \%$ (mean, 85.5\%). Total plasma TRP depletion, attributable to the combination low-TRP diet/amino acid mixture, thus ranged from $82 \%$ to $94 \%$ (mean, $89 \%$ ). Repeated measures ANOVA test for change in plasma TRP over time (baseline to hour 6 after amino acid drink) confirmed a highly significant effect $(F=171.6, d f=6, p<$ $.0001)$.

\section{Effect of TRP Depletion as Measured by CSF TRP Levels}

CSF concentrations of TRP from all time points are seen in Figure 2. Robust decreases $(F=85.8, d f=24, p<$ .0001 ) were seen in all five subjects in the $8.5-\mathrm{hr}$ interval following the amino acid drink, with $88 \%$ to $96 \%$ (mean, 92\%) depletion occurring 7 to 10 (mean \pm SD, $8 \pm 1.2$ ) hours after the amino acid mixture. As no cor-

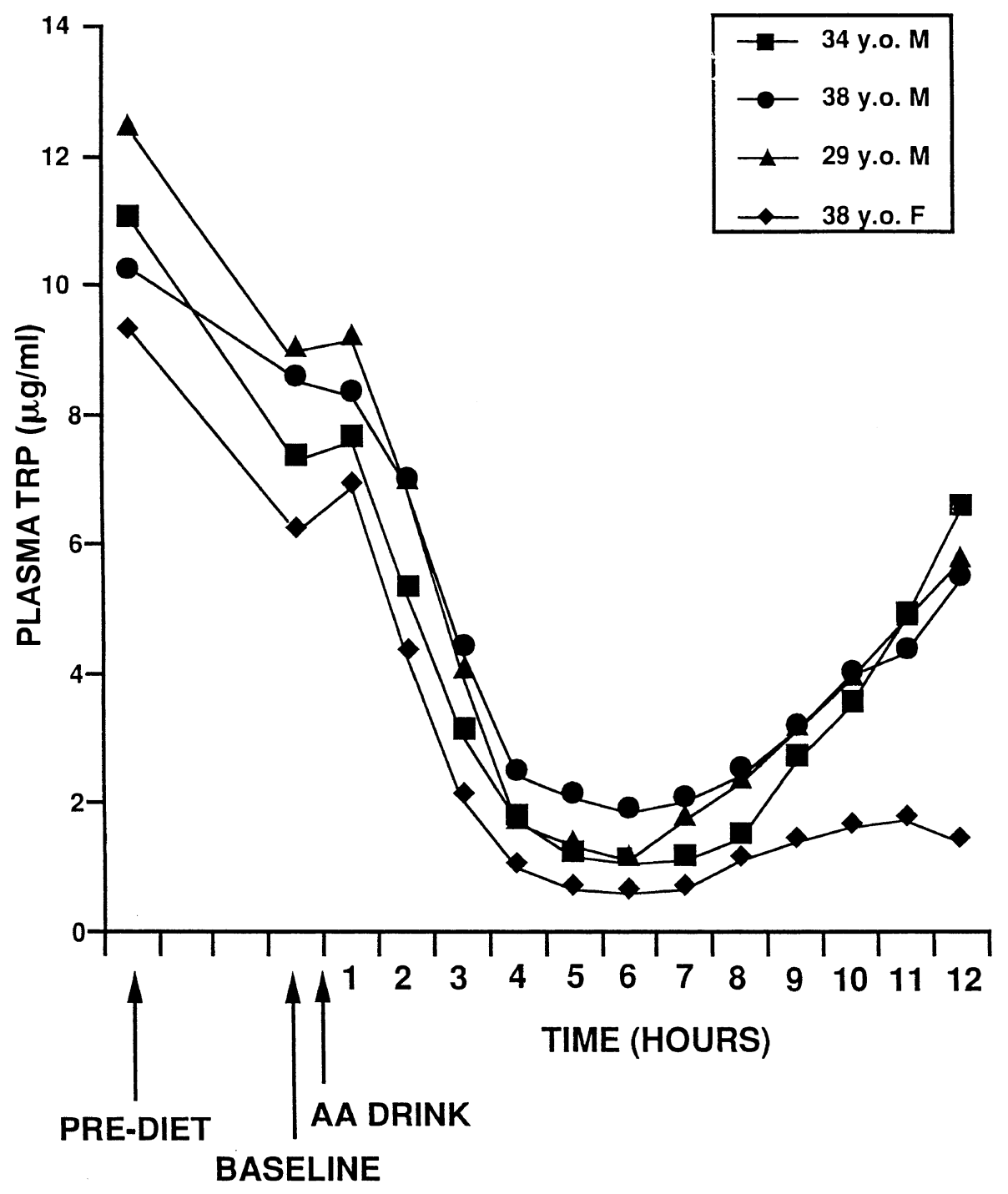

Figure 1. Plasma tryptophan concentrations in four healthy adults. Data represent a single pre-diet sample, an average of two baseline samples, and four values averaged each hour after ingestion of the amino acid drink. 


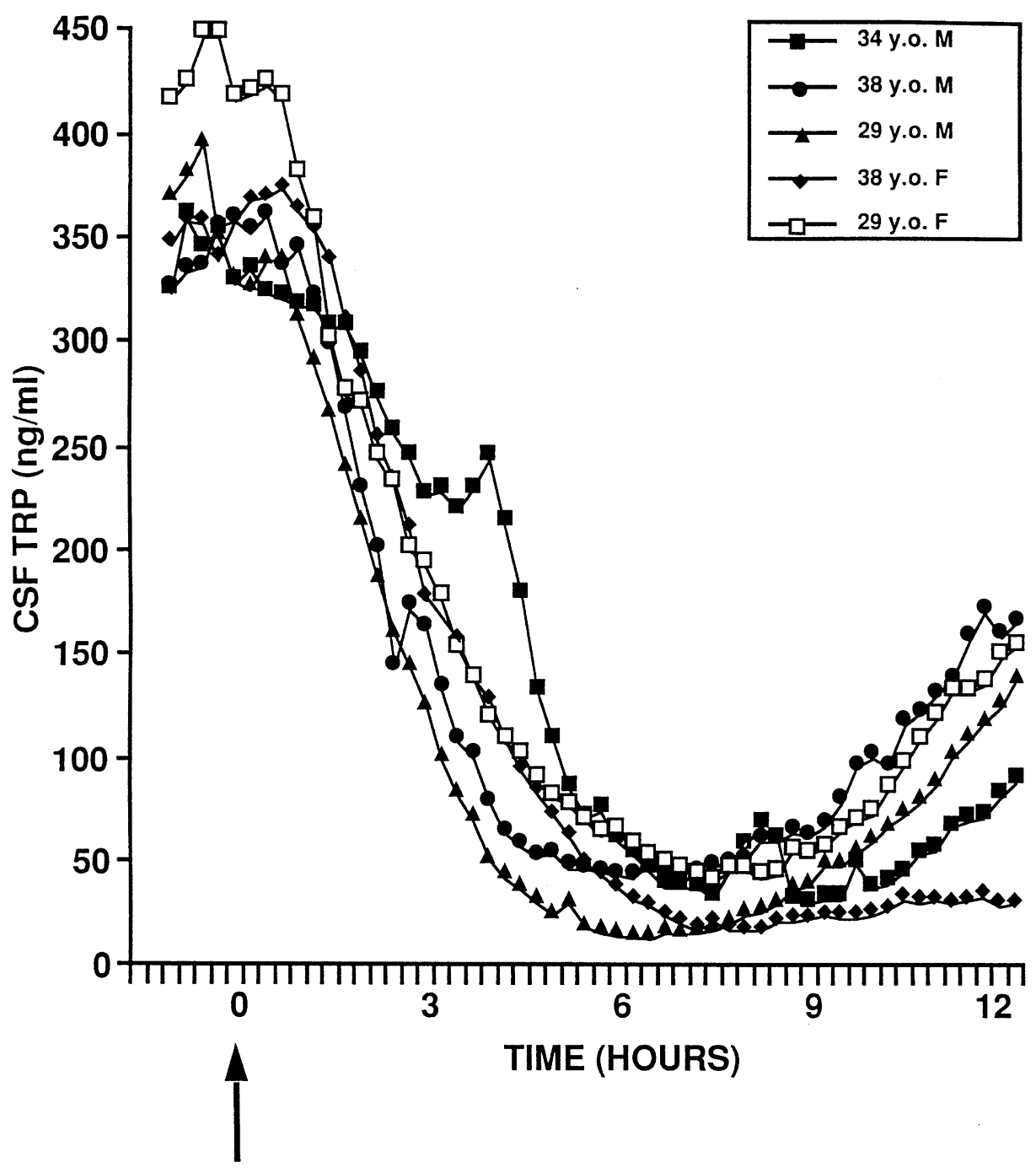

Figure 2. CSF tryptophan concentrations in five healthy adults. Values obtained from CSF fractions collected every 15 minutes during the 13.5-hour sampling period are plotted for each subject.

\section{AA DRINK}

responding pre-diet CSF sample was obtained, the total depletion of CSF TRP due to the combination of 1-day diet and the amino acid mixture was not calculable.

\section{Relationship between Plasma TRP and CSF TRP}

CSF and plasma TRP values were generally highly correlated, yet the relationship between the two measures varied somewhat across individuals with regard to time lag between plasma and CSF changes, and the rate of repletion during continued fasting. Correlation coefficients were $r=0.48(p=.01), r=0.98(p<.0001), r=$ $0.92(p<.0001)$, and $r=0.88(p<.0001)$ for the first four subjects, respectively.

\section{Effects of TRP Depletion as Measured by CSF 5-HIAA}

CSF 5-HIAA data are graphed in Figure 3. A significant, but more modest decrease $(F=9.0, d f=34, p<.0001)$, was observed in this major metabolite of 5-HT when values in the 8.5-hr interval following depletion were analyzed. Nadir values ranged from $24 \%$ to $40 \%$ of baseline; the average drop for the five subjects was $31 \%$. Lowest CSF 5-HIAA concentrations were reached no sooner than eight hours after the drink, with values continuing to trend downward even at hour 12 in several subjects.

\section{Effects of Tyrosine Load as Measured by CSF Tyrosine and HVA}

The relative tyrosine load $(6.9 \mathrm{gm})$, provided by the amino acid mixture was reflected in CSF levels of tyrosine, which rose significantly over time $(F=5.5, d f=$ $24, p<.0001$ ). As shown in Figure 4 (top), CSF tyrosine levels peaked while CSF TRP levels were at their nadir. Despite the significant increase in CSF tyrosine, concentrations of the major dopamine metabolite, HVA, did not significantly increase during the 8.5-hr interval fol- 


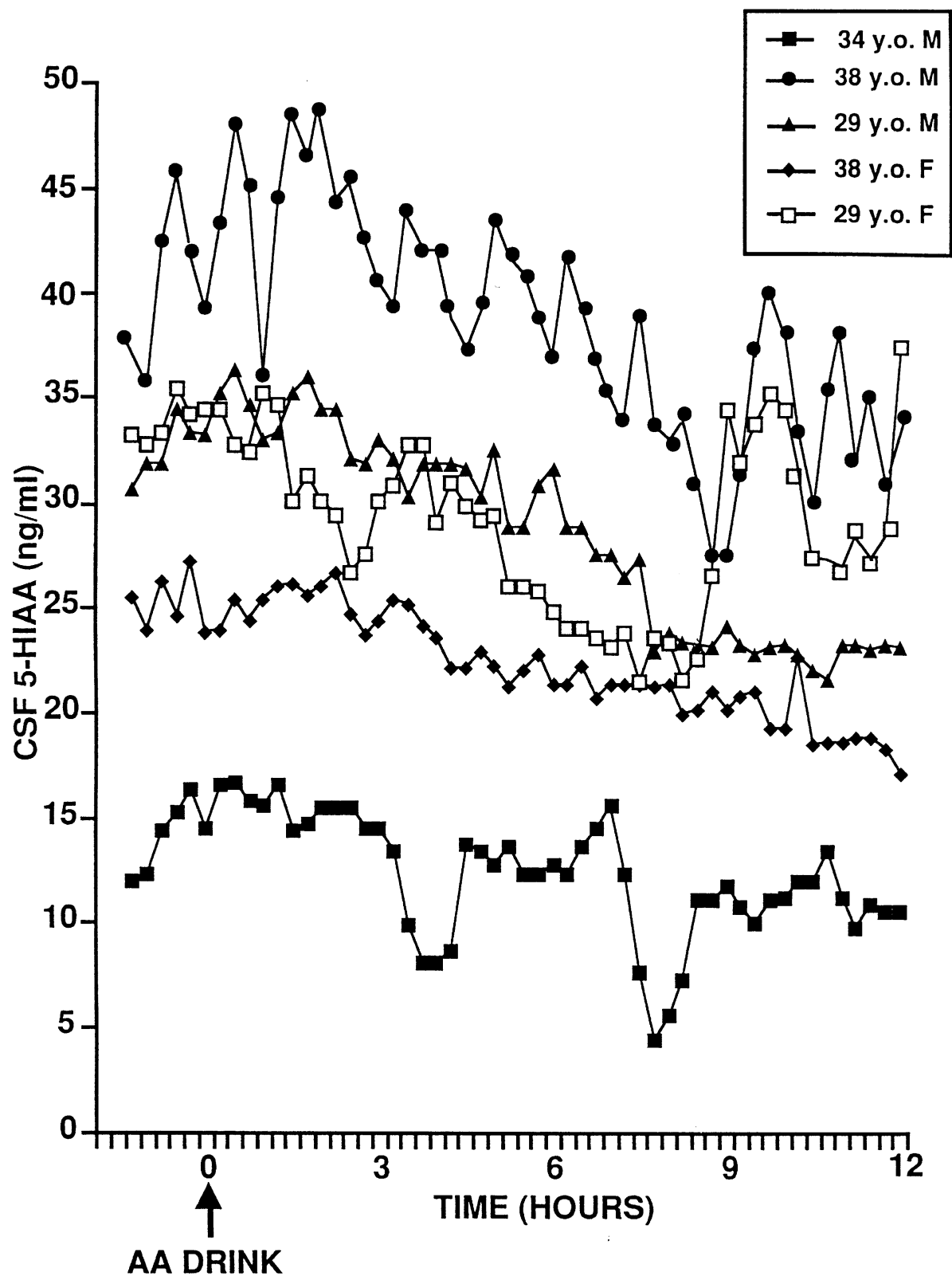

Figure 3. CSF 5-HIAA concentrations in five healthy adults. Values obtained from CSF fractions collected every 15 minutes during the 13.5-hour sampling period are plotted for each subject.

lowing depletion $(F=1.1, d f=34, p<.392)$ (Figure 4, bottom).

\section{Relationship between TRP and Tyrosine in Plasma and CSF}

Though not represented graphically here, analyses performed on data from the first subject confirmed a reciprocal relationship between TRP and tyrosine, as would be suggested by the hypothesized mechanism of the TRP depletion paradigm. The inverse relationship of the two competing amino acids was reflected in significant, negative correlation coefficients for the two compounds in both plasma $(r=0.65, p=.001)$ and CSF $(r=$ $0.86, p<.0001)$.

\section{Mood Effects Following TRP Depletion}

No significant changes were detected in repeated selfand clinician-rated measures of mood and anxiety over time, when data collected from before the diet, before the amino acid mixture, and at regular 4-hour intervals after ingestion of the mixture, were analyzed. All five subjects consistently denied symptoms of depression or anxiety during the protocol.

\section{DISCUSSION}

The issue of generalizability of results is an important one when a widely-applied, standardized research method such as oral TRP depletion is merged with an 

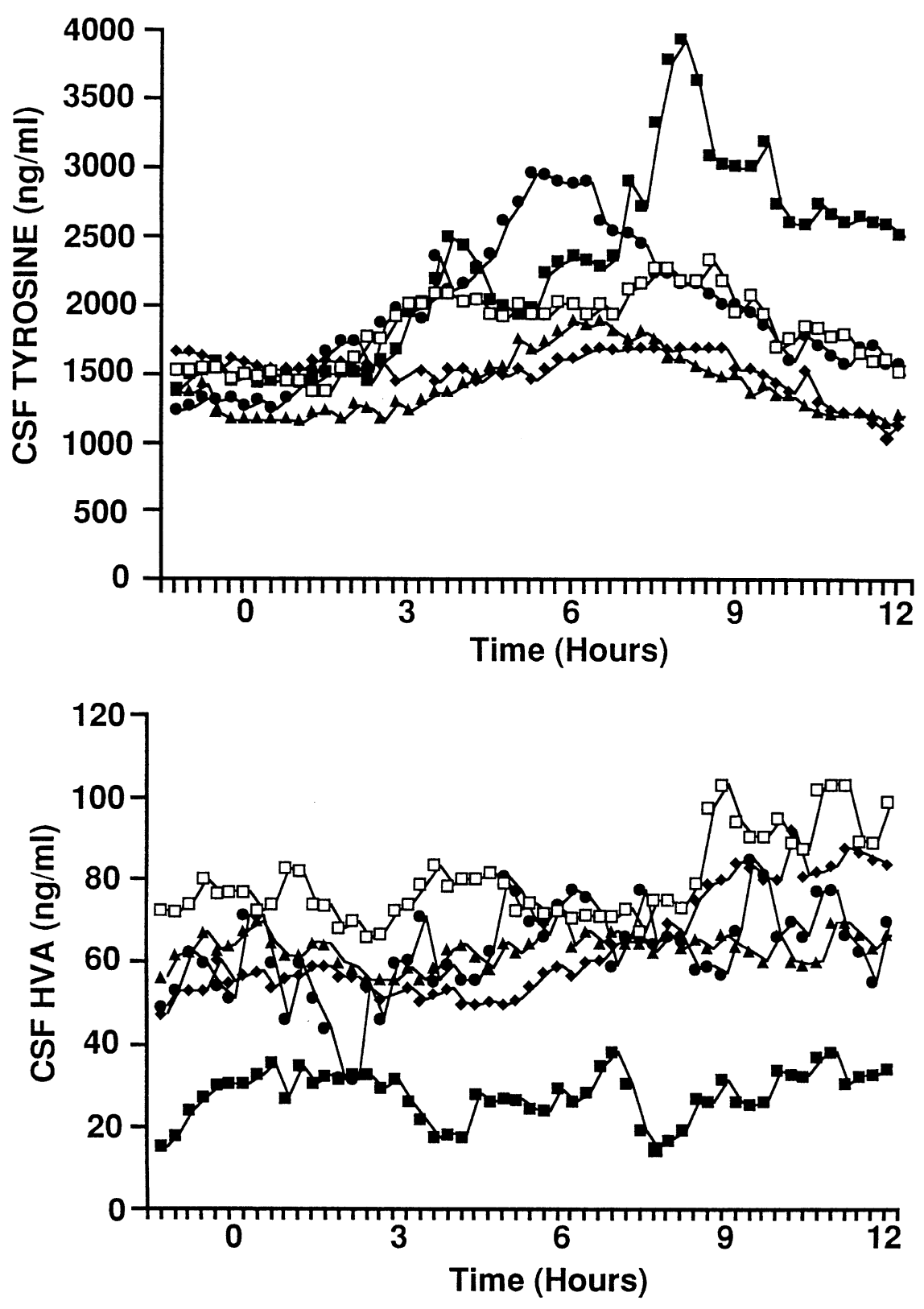

Figure 4. Top: CSF tyrosine concentrations, from fractions obtained every 15 minutes during the 13.5-hour sampling period. Bottom: CSF HVA concentrations, from the same samples. invasive procedure like lumbar puncture and spinal fluid removal. With respect to time course and degree of depletion, as measured by peripheral TRP levels, this investigation produced findings consistent with those achieved by other studies employing the combined low-TRP diet/TRP-free amino acid mixture method. Maximal effects were uniformly seen six hours after the drink was administered, an outcome similar, in time-tonadir and in magnitude of maximal depletion, to that noted by others using this method (Delgado et al. 1990, 1994). While some investigators have described a mild transient lowering of mood in normal subjects after TRP depletion (Young et al. 1995; Smith et al. 1987), a number of others have reported no significant mood effects (Abbott et al. 1992; Park et al. 1994; Moreno et al.
1995; Weltzin et al. 1995). Aside from mild fatigue attributed to prolonged bedrest, our normal subjects did not report or demonstrate the emergence of dysphoria or other depressive symptoms. Further, they denied additional stress in the form of pain, discomfort, or anxiety related to the indwelling catheter and removal of CSF during the TRP depletion procedure.

We are, thus, confident that the introduction of the CSF sampling procedure as described here did not significantly alter the biological effects of the TRP depletion paradigm as it is typically executed in human subjects.

Our research group previously used this same method to study unmanipulated (i.e., without TRP depletion) levels of CSF TRP and 5-HIAA over a 30-hour 
period in four healthy human females (Kirwin et al., 1997). The data obtained by that investigation essentially serve as control data for this study, demonstrating that CSF levels of TRP and 5-HIAA are not subject to diurnal variation or effects of chronic sampling. In Kirwin's series, CSF TRP values appeared to fluctuate in the $250-400 \mathrm{ng} / \mathrm{ml}$ range during the entire 30-hour period of sampling, a pattern dramatically different than the CSF TRP levels we observed falling into the 10-60 $\mathrm{ng} / \mathrm{ml}$ range at seven hours after ingestion of the amino acid mixture. CSF values of 5-HIAA for the controls showed no statistical rhythmicity or change over time in the period of sampling from noon to 12 midnight.

The present study provides the first confirmation that the widely-employed method of acute TRP depletion does indeed succeed in robustly diminishing central levels of TRP in humans. However, despite large declines in amino acid precursor availability, the CSF concentrations of 5-HIAA we measured suggest only a moderate (24$40 \%$ ) diminution in 5-HT metabolism. Similar reductions after TRP depletion have been reported for 5-HIAA levels in CSF of vervet monkeys (Young et al. 1989), and in cortical dialysate of rats (Heslop et al. 1991). Other studies of brain or dialysate 5-HT and 5-HIAA levels (Trulson 1985; Moja et al. 1989; Bel and Artigas 1996) suggest that, in rats, TRP depletion can produce 5-HT and 5-HIAA reductions of greater than $50 \%$.

Assuming the $24-40 \%$ reductions seen here for human CSF 5-HIAA accurately index the diminution in brain 5-HT turnover, the critical question remains: How closely does this decrease reflect changes in central 5-HT functioning? In general, the available animal studies examining both 5-HT and 5-HIAA after TRP depletion indicate the two compounds were similarly reduced (e.g., 27\% 5-HT and 44\% 5-HIAA decreases reported by Trulson 1985; 41\% 5-HT and 49\% 5-HIAA decreases reported by Moja et al. 1989). Of special relevance with respect to the functional consequences of TRP depletion is an in-vivo dialysis study of 5-HT release in rat hippocampus (Gartside et al. 1992). There, decreased TRP availability was shown to substantially $(\sim 50 \%)$ reduce electrically-evoked releases of $5-\mathrm{HT}$. Based on these studies and on our human CSF data, it can be suggested that the standard TRP depletion protocol produces a significant, but not marked, decline in central 5-HT turnover and function. This tentative conclusion stands in sharp contrast to the recently reported results of a PET study that examined human brain 5-HT turnover by measuring accumulation of $\alpha$-methyl 5-HT after $\alpha$-methyl TRP administration (Nishizawa et al. 1997). There, a marked lowering of brain 5-HT synthesis in all brain regions was seen following acute TRP depletion, with reductions in the rate of 5-HT synthesis by a factor of about 9.5 in males and of about 40 in females. Some of the apparent discrepancy between the findings of Nishizawa's group and those of the present study could be due to the use of a measure reflecting $5-\mathrm{HT}$ synthesis rather than 5-HT catabolism. However, it remains difficult to understand how 5-HT synthesis could decline so substantially following acute TRP depletion without corresponding marked declines in 5-HIAA.

The hypothesized method of action for acute plasma and central TRP depletion is supported by reciprocal changes we measured in TRP and tyrosine. Consistent with the notion of protein synthesis induction, the relative tyrosine load $(6.9 \mathrm{gm})$ was reflected by a lowering of the plasma TRP: tyrosine ratio as expected and previously demonstrated (Perez-Cruet et al. 1974). Concurrent CSF sampling afforded a new opportunity to observe this same, reciprocal relationship unfold at a central level, supporting the proposed mechanism of competitive, active transport of these large neutral amino acids across the blood-brain barrier. Measurements of CSF HVA allowed us to explore the possibility that the increases in tyrosine might increase catecholamine synthesis. This hypothesis was not supported by results of repeated-measures ANOVA for CSF HVA, which did not show a significant pattern of change during the interval following depletion, despite the substantial rise in the precursor tyrosine.

Our data demonstrated significant decreases in CSF 5-HIAA over the 8-12 hour period following administration of the amino acid drink, but no corresponding behavioral changes were noted in five healthy subjects. Perhaps a certain threshold or magnitude of 5-HT depletion, unobtained in the present study, is necessary to produce the clinical correlates of compromised 5-HT function that have been described in various psychiatric populations undergoing acute TRP depletion. A nextstep would include the replication of this protocol with a population of patients with major depression, recently remitted on selective serotonin reuptake inhibitors (SSRIs). Using the continuous sampling method to compare baseline levels and dynamic changes in CSF 5HIAA between these healthy controls and patients experiencing depressive relapse might further our understanding of the 5-HT dysregulation underlying depression and the mechanism of action of drugs which treat it.

Continuous sampling of CSF samples during acute TRP depletion offers a novel approach to the assessment of serotonergic function in the human brain. However, due to antomical and metabolic factors, 5-HIAA dynamics measured in lumbar CSF can only approximate changes in central 5-HT release and function. Unfortunately, it appears that direct measurements of 5-HT in human CSF with currently available methods are not informative (Anderson et al. 1990), and determinations in human extracellular fluid are not practical. Given these caveats, the present data suggest that acute TRP depletion does robustly lower central TRP levels, modestly lowers central 5-HIAA levels, and may reduce general 5-HT function. 


\section{ACKNOWLEDGMENTS}

This work was supported in part by MHCRC \#30292, NARSAD Young Investigator Awards (Drs. Carpenter, McDougle, and Pelton), and by the Department of Mental Health and Addiction Services, State of Connecticut. The clinical, research, and pharmacy staffs of the Clinical Neuroscience Research Unit provided expert assistance. Laura Hall, M.S. provided laboratory assistance; Sally Vegso, M.S. assisted in data analysis and management; Kathryn Czarkowski, B.S. prepared graphs; and Betsy Kyle assisted in manuscript preparation. Special thanks go to Fern Severino, M.D. of the Yale University School of Medicine Department of Anesthesia, for expert consultation and supervision of catheter placements.

\section{REFERENCES}

Abbott F, Etienne P, Franklin K, Morgan M, Sewitch M, Young S (1992): Acute tryptophan depletion blocks morphine analgesia in the cold-pressor test in humans. Psychopharmacology 108:60-66

Anderson GM, Feibel FC, Cohen DJ (1987): Determination of serotonin in whole blood, platelet-rich plasma, plateletpoor plasma, and plasma ultrafiltrate. Life Sci 40:10631070

Anderson GM, Mefford IN, Tolliver TJ, Riddle MA, Ocame DM, Leckman JF, Cohen DJ (1990): Serotonin in human lumbar cerebrospinal fluid: A reassessment. Life Sci 46:247-255

Anderson GM, Young JG, Cohen DJ (1979): A rapid liquid chromatographic method for the determination of tyrosine, tryptophan, 5-hydroxyindoleacetic acid and homovanillic acid in cerebrospinal fluid. J Chrom 142:501-505

Beck A, Ward C, Mendelson M, Mock J, Erbaugh J (1961): An inventory for measuring depression. Arch Gen Psychiatr 4:53-63

Bel N, Artigas F (1996): Reduction of serotonergic function in rat brain by tryptophan depletion: Effects in control and fluvoxamine-treated rats. J Neurochem 67:669-676

Biggio G, Fadda F, Fanni P, Tagliamonte A, Gessa G (1974): Rapid depletion of serum tryptophan, brain tryptophan, serotonin and 5-hydroxyindoleacetic acid by a tryptophan-free diet. Life Sci 14:1321-1329

Bruce JN, Oldfield EH (1988): Method for sequential sampling of cerebrospinal fluid in humans. Neurosurgery 23:788-790

Clemens J, Bennett D, Fuller R (1980): The effect of a tryptophan-free diet on prolactin and corticosterone release by serotonergic stimuli. Hormone Metab Res 12:35-38

Delgado PL, Charney DS, Price LH, Aghajanian GK, Landis H, Heninger GR (1990): Serotonin function and the mechanism of antidepressant action: Reversal of antidepressant induced remission by rapid depletion of plasma tryptophan. Arch Gen Psychiatr 47:411-418

Delgado PL, Charney DS, Price LH, Landis H, Heninger GR (1989): Neuroendocrine and behavioral effects of dietary tryptophan depletion in healthy subjects. Life Sci 45:2323-2332

Delgado PL, Price LH, Miller HL, Salomon RM, Aghajanian
GK, Heninger GR, Charney DS (1994): Serotonin and the neurobiology of depression: Effects of tryptophan depletion in drug-free depressed patients. Arch Gen Psychiatr 51:865-874

Fernstrom JD (1977): Effects of the diet on brain neurotransmitters. Metabolism 26:207-223

Gartside S, Cowen P, Sharp T (1992): Evidence that the large neutral amino acid L-valine decreases electrically-evoked release of 5-HT in rat hippocampus. Psychopharmacology 109:251-253

Geracioti TD, Orth DN, Ekhator NN, Blumenkopf B, Loosen PT (1992): Serial cerebrospinal fluid corticotrophin-releasing hormone concentrations in healthy and depressed humans. J Clin Endocrinol Metab 74:1325-1330

Geracioti TD, Schmidt D, Ekhator NN, Shelton R, Parris W, Loosen PT, Ebert MH (1993): Cerebrospinal fluid norepinephrine concentrations and dynamics in depressed patients and normal controls. Depression 1:149-155

Gessa GL, Biggio G, Fadda F, Corsini GV, Tagliamonte A (1974): Effect of the oral administration of tryptophan-free amino acid mixture on serum tryptophan, brain tryptophan and serotonin metabolism. J Neurochem 22:869870

Gibbons JL, Barr GA, Bridger WH, Leibowitz SF (1979): Manipulations of dietary tryptophan: Effects on mouse killing and brain serotonin in the rat. Brain Res 169:139-153

Hamilton M (1959): The assessment of anxiety states by rating. Br J Med Psychol 32:50-55

Harper A, Benevenga N, Wohlhueter R (1970): Effects of ingestion of disproportionate amounts of amino acids. Physiol Rev 50:428-548

Heslop K, Portas CM, Curzon G (1991): Effect of altered tryptophan availability on tissue and extracellular serotonin in the rat cortex. In Rollema $\mathrm{H}$, Westerink BHC, Drijfhout WJ (eds), Monitoring Molecules in Neurosciences. Proceedings of the 5th International Conference on In Vivo Methods. Groningen, The Netherlands, University Centre for Pharmacy, pp 259-260

Kirwin PD, Anderson GM, Chappell PB, Saberski L, Leckman JF, Geracioti TD, Heninger GR, Price LH, McDougle CJ (1997): Assessment of diurnal variation of cerebrospinal fluid tryptophan and 5-hydroxyindoleacetic acid concentrations in healthy human females. Life Sci 60:899-907

Lytle LD, Messing RB, Fisher L, Phebus L (1975): Effects of long-term corn consumption on brain serotonin and the response to electric shock. Science 190:692-694.

Maes M, Meltzer H (1995): The serotonin hypothesis of major depression. In Bloom F, Kupher D (eds), Psychopharmacology: The Fourth Generation of Progress. New York, Raven Press, Ltd., pp 933-944

Mazure C, Nelson J, Price L (1986): Reliability and validity of the symptoms of major depressive illness. Arch Gen Psychiatr 43:451-456

McCormack H, Horne D, Sheather S (1988): Clinical applications of visual analogue scales: A critical review. Psychol Med 18:1007-1019

Messing RB, Fisher LA, Phebus L, Lytle LD (1976): Interaction of diet and drugs in the regulation of brain 5-hydroxyindoles and the response to painful electric shock. Life Sci 18:707-714 
Miller HL, Delgado PL, Salomon RM, Licinio J, Barr LC, Charney DS (1992): Acute tryptophan depletion: A method of studying antidepressant action. J Clin Psychiatr 53 (Suppl 10):28-35

Moja EA, Cipollo P, Castoldi D, Tofanetti O (1989): Doseresponse decrease in plasma tryptophan and in brain tryptophan and serotonin after tryptophan-free amino acid mixtures in rats. Life Sci 44:971-976

Moja EA, Mendelson WB, Stoff DM, Gillin JC, Wyatt RJ (1979): Reduction of REM sleep by a tryptophan-free amino acid diet. Life Sci 24:1467-1470

Moja EA, Stoff DM, Gessa GL, Castoldi D, Assereto R, Tofanetti O (1988): Decrease in plasma tryptophan after tryptophan-free amino acid mixtures in man. Life Sci 42:1551-1556

Moreno F, Strayer L, Heninger G, Delgado P (1995): Mood response to tryptophan depletion and vulnerability to depression. Soc Neurosci 21:194

Nishizawa S, Benkelfat C, Young S, Leyton S, Mzengeza S, DeMontigny C, Blier P, Diksic M (1997): Differences between males and females in rates of serotonin synthesis in human brain. Proc Nat Acad Sci USA 94:53085313

Park SB, Coull JT, McShane RH, Young AH, Sahakian BJ, Robbins TW, Cowen PJ (1994): Tryptophan depletion in normal volunteers produces selective impairments in learning and memory. Neuropharmacology 33:575-588

Perez-Cruet J, Chase TN, Murphy DL (1974): Dietary regulation of brain tryptophan metabolism by plasma ratio of free tryptophan and neutral amino acids in humans. Nature 248:693-695

Price L, Malison R, McDougle C, Pelton G, Heninger G (1997a): The neurobiology of tryptophan depletion in depression: Effects of intravenous tryptophan infusion. Biol Psychiatr, in press

Price L, Malison R, McDougle C, McCance-Kratz E, Owen K,
Heninger G (1997b): The neurobiology of tryptophan depletion in depression: Effects of m-chlorophenylpiperazine (mCPP). Neuropsychopharmacology 17:246257

Schaechter JD, Wurtman RJ (1990): Serotonin release varies with brain tryptophan levels. Brain Res 532:203-210

Smith S, Pihl R, Young S, Ervin F (1987): A test of possible cognitive and environmental influences on the mood lowering effect of tryptophan depletion in normal males. Psychopharmacology 91:451-457

Trulson M (1985): Dietary tryptophan does not alter the function of brain serotonin neurons. Life Sci 37:10671072

Walters JK, Davis M, Sheard MH (1979): Tryptophan-free diet: Effects on the acoustic startle reflex in rats. Psychopharmacology 62:103-109

Weltzin TE, Fernstrom MH, Fernstrom JD, Neuberger SK, Kaye WH (1995): Acute tryptophan depletion and increased food intake and irritability in bulimia nervosa. Am J Psychiatr 152:1668-1671

Woods S, Charney D, Loke J, Goodman W, Redman D, Heninger G (1986): Carbon dioxine sensitivity in panic anxiety; ventilatory and anxiogenic response to $\mathrm{CO} 2$ in healthy subjects and panic disorder patients before and after alprazolam treatment. Arch Gen Psychiatr 43:900909

Young S (1991): Some effects of dietary components (amino acids, carbohydrate, folic acid) on brain serotonin synthesis, mood, and behavior. Canadian J Physiol Pharmacol 69:893-903

Young SN, Ervin FR, Pihl RO, Finn P (1989): Biochemical aspects of tryptophan depletion in primates. Psychopharmacology 98:508-511

Young SN, Smith SE, Pihl RD, Ervin FR (1995): Tryptophan depletion causes a rapid lowering of mood in normal males. Psychopharmacology (Berlin) 87:173-177 